\title{
Goal Oriented Personalisation with SCORM
}

\author{
Guillermo Power ${ }^{1}$, Hugh C Davis ${ }^{1}$, Alexandra I. Cristea ${ }^{2}$, Craig Stewart ${ }^{3}$ and Helen Ashman ${ }^{3}$ \\ ${ }^{1}$ Learning Technologies Group, ECS, University Of Southampton, Southampton, UK \\ +44(0)238059 8867, \{gp21hcd\}@ecs.soton.ac.uk \\ ${ }^{2}$ Faculty of Computer Science and Mathematics, Eindhoven University of Technology, \\ P.O. 513, 5600 MB Eindhoven, The Netherlands,+31-40-2474350, a.i.cristea@tue.nl \\ ${ }^{3}$ School of Computer Science and Information Technology, University of Nottingham, \\ Nottingham, UK, +44(0)115846 6505, \{cds|hla\}@cs.nott.ac.uk
}

\begin{abstract}
This paper presents an innovative approach to personalize on-line content to the needs of individual learners. We use a regular educational environment, the Blackboard ${ }^{T M}$ Learning Management System, with a new approach: we add adaptivity and personalization to it by means of authoring the goaloriented material in an Adaptive Hypermedia authoring system, MOT, and delivering it in Blackboard via a conversion to the SCORM specification. This represents the first attempt to connect Adaptive Hypermedia and Learning Management Systems.
\end{abstract}

\section{Introduction}

This paper describes an approach to personalising on-line materials to the needs of individual learners in order to improve the suitability of the materials to the users' goals and current understanding so that the time spent on the current task is more focussed. In this introduction we explain the particular context in which we are experimenting with this technology.

At the University of Southampton we teach a final year undergraduate elective course on Hypertext and Web Technology. One third of this unit, which covers research issues in Hypertext, is carried out in the summer term at the same time as the students are finishing their major individual project. Inevitably they tend to prioritise their project work and attendance at lectures is poor. For the purposes of this unit we have decided to accept the students' behaviour and to organise this piece of the unit so that the students may carry out all the required work on line and at their preferred time.
This part of the unit is therefore presented as a set of on-line materials (papers from the Hypertext literature) that the students are expected to access, along with some commentaries by the lecturer (which will be the basis of the voluntary lectures). The students are given six questions (goals) which they are required to answer in order to prepare for the examination. The examination is held on computers which are on-line and students are allowed access to the literature, their notes and any other static materials available on the Web. The questions will be variations of the questions they have been asked to prepare, and the examination is entirely concerned with testing student's ability to analyse, evaluate and synthesise and in no part concerned with simple recall of facts.

The body of literature the students are given is far too large for a single student to read properly in the time available (about 50 papers in 4 weeks). This is deliberate - the desired learning outcomes are that the students learn to scan read and that they learn to work together to conquer large learning problems. To this end students are encouraged to use the student WIKI to communicate what they have learned and to hold online or face-to-face meetings to discuss their understanding. Some students participate in this collaborative phase and others do not.

We decided to look at what tools we might provide to help a student to read at the detail they choose and to locate particular passages - what we call Goal Oriented Personalisation. Students might skim read, or read all the materials available. Since we were also involved in a project on authoring adaptive materials (ADAPT [2]) we decided to use this course as an experiment in the application of our emerging solutions.

To this end we tagged the reading materials with information that allows the system to produce a 
personalised lesson which responds to one of a predefined set of goals. As this is a real course we did not wish to experiment with untested delivery platforms. Instead we wished to deliver through the usual platforms - in our case we have an Intranet website and Blackboard $^{\mathrm{TM}}$. The preferred solution was to deliver the personalised lesson as a SCORM content package that could be accessed via the Blackboard Learning Resource iNterchange (LRN) viewer or via a third party such as ADL's SCORM [3] viewer.

Neither SCORM nor Blackboard offer the ability to alter the presentation of content to the goals of a user. Therefore we needed to arrange for a pre- personalised SCORM content package (previously created by the authoring system) to be available for each user.

For this purpose, we started with an Adaptive Hypermedia Authoring system, MOT [11], which is able to provide the adaptive specification for our course. The material created in MOT is then transformed into the SCORM specification.

The remainder of this paper describes the theory and technology we used to solve this problem and achieve these personalised lessons

\section{Our Approach}

We had two main requirements in terms of input and output; firstly the user had to be provided with a easy to use, intuitive interface to help him/her generate and edit personalizable learning content. Secondly we had to be able to display the SCORM output in a widely available Virtual Learning Environment.

As there aren't any standards for describing personalized hypermedia learning material, there wasn't an obvious format for us to choose for our input data, however, we required a tool that allowed a teacher to edit and create an adaptive content easily. In MOT we found a tool that fulfilled this criteria; MOT provides the author with an easy to use web interface that allows for the creation of adaptive learning material.

With MOT, the subject matter of the course to be designed can be modeled by means of concept maps. Based on these concept maps lessons can be constructed. MOT provides the user with an intuitive Web interface to edit both Concept Maps and Lessons.

Secondly our choice of Virtual Learning Environment was clear from the start, the University of Southampton uses the Blackboard Virtual Learning Environment to distribute learning material to students. Given that the latest versions of it support SCORM it was a clear choice that we should test out output using Blackboard.

\section{Background to MOT}

MOT [11] is a generic Adaptive Hypermedia System web-authoring environment developed at the Eindhoven University of Technology (TU/e), constructed based on MyET [8], LAOS (Layered WWW AHS Authoring Model with its corresponding Algebraic Operators) [7] and LAG (Layers of Adaptive Granulation Model) [4]. MOT implements LAOS by supporting a domain model, in the form of a conceptual hierarchical layer (of atomic and composite concepts, built of a number of attributes), and a goal and constraints model, in the form of a lesson layer, dealing with alternative presentation of contents at attribute level or above. This structure conforms to the requirements of $\mathrm{W} 3 \mathrm{C}$ towards the third generation Web, called the Semantic Web[14]. MOT implements LAG, by having an adaptation model with three possible input levels for adaptation functionality. The adaptation itself follows a three-layer granularity structure, of direct adaptation techniques and rules, an adaptation language and adaptation strategies.

For the purpose of this paper we concentrate on the domain and lesson models. In the following, we give more detail of the layers that we used in this example.

\subsection{Concepts (Domain Layer) in MOT}

The MOT Domain Layer contains one or more subconcepts, which are in turn concepts themselves. MOT calls a collection of such constructs a conceptmap. Each concept in a conceptmap is described by a number of concept attributes; these hold pieces of information about the concept they belong to. There are several kinds of attributes, for example, a concept can have a title, description, text, etc. Concept attributes can be related to each other. Such relations between concepts indicate that their attributes treat similar topics

\subsection{Lessons (Goal \& Constraints Layer) in MOT}

The Goal \& Constraint Layer in MOT is represented by lessons. A lesson can contain sublessons, which are lessons in their own right. This hierarchical structure of lessons is connected via AND or OR connectors. A lesson contains, besides the sublesson holders, one or more concept attributes, which are also AND- or OR-connected. The purpose of this layer is to collect discrete pieces of information (concept attributes) from multiple Domain Maps, and to fit them together in a suitable manner for presentation (order, importance, etc.) to the student. 


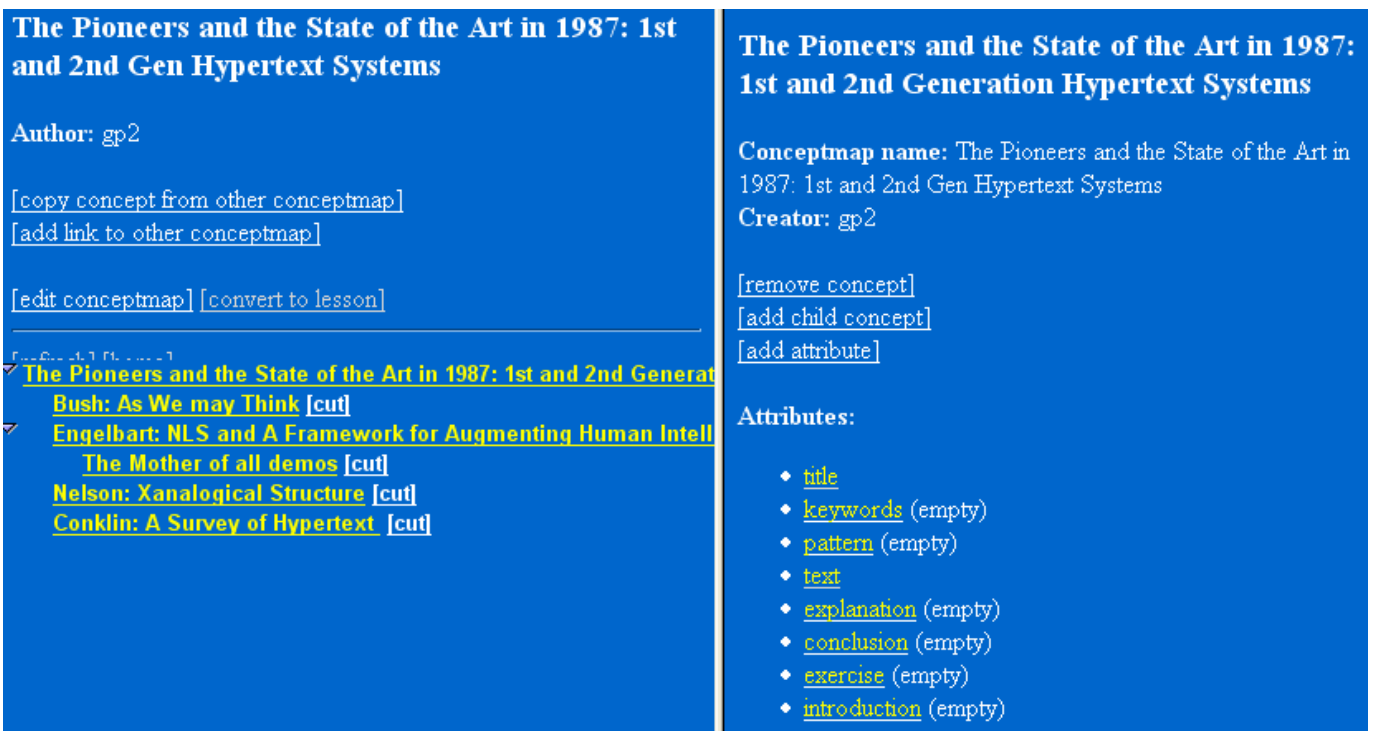

Figure 1: Domain (Concepts) Layer Interface

\subsection{MOT Implementation of a New Course}

We choose MOT as our system to design adaptive content because it is a powerful Adaptive Hypermedia System design tool. MOT presents the user with a webbased interface that allows the author to design adaptive content. The interface is divided into three sections:

1. The Domain Layer Interface, which allows users to design conceptmaps (see Figure 1 for a glimpse at the Southampton course written in MOT).

2. The Goal \& Constraints Layer Interface, which allows users to design lessons (see Figure 2 for a partially transformed Southampton course in the MOT Goal \& Constraints Layer).

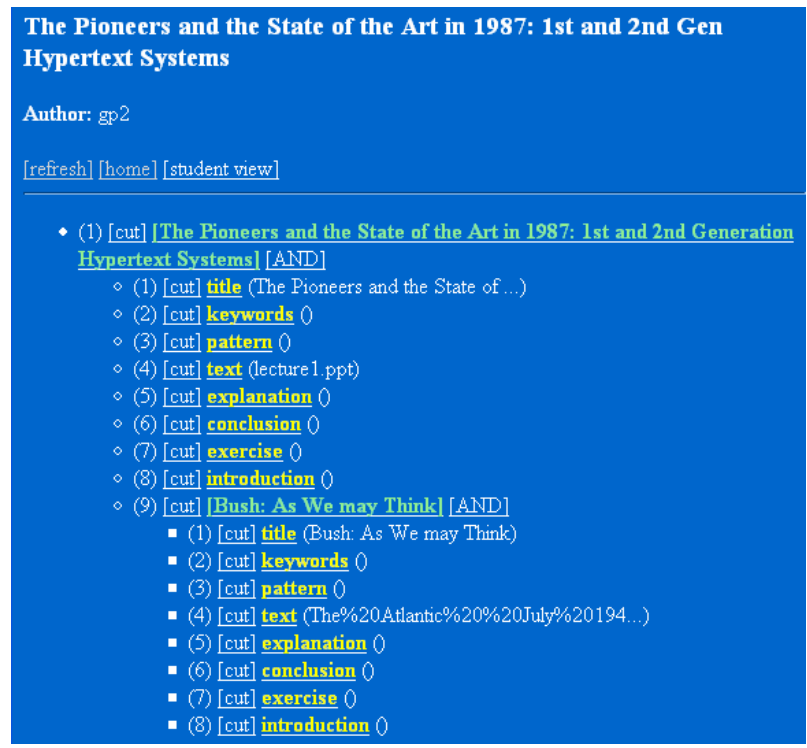

Figure 2: Goal-Constraints (Lessons) Layer
3. Finally the Adaptation Model Interface, which consists of an adaptation language, allowing the user to define different adaptation strategies.

For the purposes of this paper we are ignoring this last layer as we are defining our own separate adaptation strategy, coupled to the conversion program.

\subsection{The MOT Database}

MOT stores its data in a MySQL database. This database uses several pointers to represent the tree structures, seen in the concept and lesson layers, on the two dimensional relational database.

These pointers are unique identifiers within the database that we have opted to employ as unique identifiers within the SCORM manifest created by the MOT to SCORM converter, as shown in Figure 3. These pointers are unique identifiers within the database that we have opted to employ as unique identifiers within the SCORM manifest created by the MOT to SCORM converter, as shown in Figure 3.

\section{MOT-to-SCORM converter}

We have developed a converter that will take a MOT lesson such as the one described in the previous section and generate a lesson in SCORM using the lesson's AND-OR connections and concept group weights for its adaptation criteria. For the first version of the converter we choose to ignore MOT's powerful adaptation layer, because this would have significantly increased development difficulty. Therefore we have developed an initial model to tailor content to learners' differing goals and degree of interest; the adaptation strategy is hard coded into the converter. 


\subsection{Adaptation Model}

To facilitate the description of our adaptation model we present a simplified way of looking at a MOT lesson by removing the concept of sub-lessons.

The simplified lesson can be seen as a collection of concepts, with the levels representing levels on the concept tree. Each concept has a set of attributes, a connector and a weight. In MOT each lesson concept has a weight as well, but for our purposes this weight is not needed, as each concept will form an entire SCORM item.

When creating a lesson for a specific learner or group of learners, we specify a cut-off weight. If the weight is more than or equal to the cut-off and it is OR connected, then the concept is suitable for that learner. Note: all AND connected concepts will be classed as suitable for that learner. Alternatively, if a concept has a weight less than the cut-off and it is OR connected, it will be classed as unsuitable for that learner.

\subsection{Interface}

To edit the hypermedia content the author is expected to make use of the MOT editors for the Concept Layer (see Figure 1) and Domain-Goal Layer (see Figure 2) to create a lesson. Currently the MOTto-SCORM converter expects to find a lesson with the entire set of standard MOT concept attributes (title, keywords, pattern, text, explanation, conclusion, exercise and introduction), although currently we utilize only the title and the text attributes. The converter prompts the user to select a lesson to be converted and to input a cut-off value, and then it converts this lesson to SCORM creating a manifest file for it. Finally, the user is expected to create their own IMS content package [9] with the manifest and all resource files by simply creating a zip file of the manifest and the resource files.

To display the resulting adapted lesson we have used Microsoft's LRN viewer, Blackboard's LRN viewer and a third party SCORM viewer Plug-In for Blackboard (see Figure 4).

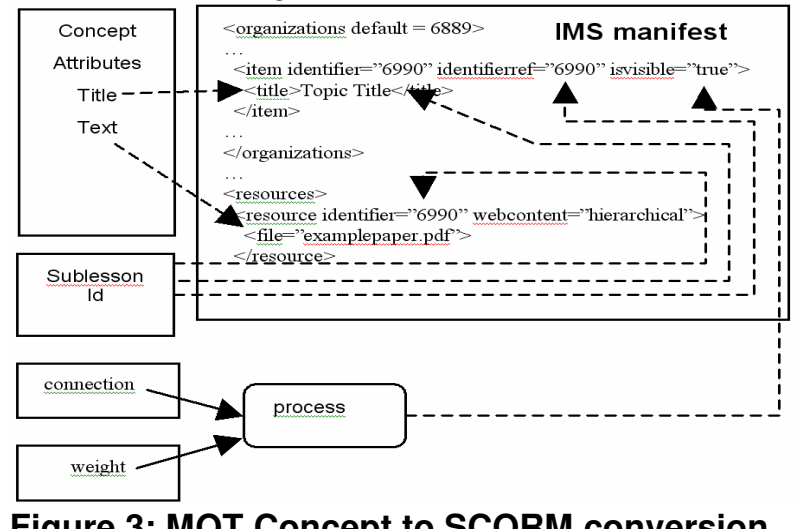

Figure 3: MOT Concept to SCORM conversion

\subsection{MOT to SCORM Conversion}

The MOT-to-SCORM converter takes one MOT lesson and converts it to a single SCORM manifest in an IMS Content Package according to the adaptation rules. Each concept in the lesson is converted to be a single item in the organization section of the manifest, using the title of that concept and the sublesson Id as a unique identifier. In our example each item has a resource file associated with it. The name for the resource file is entered in the text attribute of that concept and then it is included in the IMS content package for the course (see Figure 3)

To test the converter, we used the real world example of recommended reading material for an Advanced Hypermedia unit in a third year undergraduate course as discussed in the Introduction (see Figure 4). We recreated the original suggested reading list in MOT using AND/OR connection conditions and weights reflecting the material that was considered necessary, as well as optional material, and the degree of complexity of it.

There are two possible adaptations the software carries out:

- Modify the title so that concepts pertaining to sections unsuitable for that learner are preceded by the word "OPTIONAL" (see Figure 4).

- Secondly, the 'isvisible' attribute for each item that is not suitable for the learner is set to false (see Figure 3).

\subsection{Implementation Details}

The MOT-to-SCORM converter was coded in Java. During implementation we noticed how complex it is to write code to extract information from the hierarchical structures of the MOT database in both the Domain and Goal-Constraints layers. Therefore we have started developing a MOT Java API to facilitate the development of future Java applications using the MOT database.

\section{Testing \& Evaluation}

We have yet to conduct any quantitative evaluation of this approach, but we have carried out testing to ensure that the system provides sensible routes through the material as intended. As explained in the introduction, our Hypertext and Web Technologies unit posed the students with six questions they should be able to answer upon completing the course.

We had a collection of around 50 papers from the Hypertext literature plus the powerpoint lecture slides, and using MOT we created routes through the materials to respond to the different questions, 
personalized to a user model, in which the user decided the depth of reading they wish to undertake.

Students will be presented with these materials next semester and they will be disseminated using the SCORM Viewer building block in Blackboard.

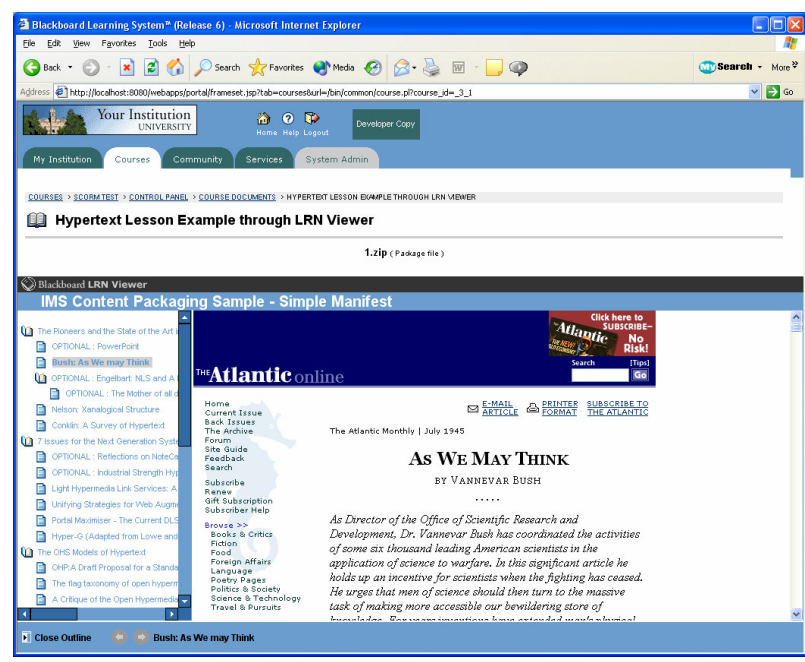

Figure 4: Adapted course shown through Blackboard's LRN viewer

\section{Discussion \& conclusions}

In this paper we have discussed how to prepare a personalised lesson for a static delivery system. Most current Adaptive Educational Hypermedia systems dynamically adapt the delivery of content to a learner's needs, be it their knowledge [4] or, more recently, to their Learning Style [5]. However these truly adaptive systems have currently progressed little beyond their research prototypes.

The contribution of this work is to demonstrate how existing materials can be pre-adapted (rather than delivered adaptively) for use in existing commercial learning environments such as Blackboard. We believe this could provide a stepping stone towards the introduction of personalised adaptive learning. As an example of this we have discussed a real problem that we solved with this new solution, the "Hypertext and Web Technology" course at the University of Southampton, and how goal oriented adaptation can be achieved within an apparently static delivery environment. The approach is similar to that taken by the Dynamic Courseware Generator [10], produces standard SCORM output, and differs from the approach taken in [1] in that the SCORM is statically generated, rather than dynamically adapted.

Finally, this paper describes yet another step towards the long term goal of synergistically interfacing many Educational Hypermedia (EH) delivery systems. The ADAPT project [2] has initiated research in this area. And represents the framework of the research presented in this paper. In ADAPT we have already used MOT as a generic authoring system, had MOT Lessons automatically converted for use in entirely unrelated delivery systems such as AHA! [12], WHURLE [13], and now Blackboard.

\section{Acknowledgments}

This work is supported by the Minerva Socrates EU project ADAPT (101144-CP-1-2002-NL-MINERVAMPP [1]).

\section{References}

[1] Abdullah, N.A., Bailey, C.P., and Davis, H.C, "Augmenting SCORM Manifest with Adaptive Links", In Proc ACM Conference on Hypertext and Hypermedia, August, 2004

[2] ADAPT project,

http://wwwis.win.tue.nl/ acristea/HTML/Minerva/index.html [3] ADL, SCORM,

http://www.adlnet.org/index.cfm?fuseaction=scormabt

[4] Brusilovsky, P. Adaptive hypermedia. User Modeling and User Adapted Interaction,11(1/2), (2001), 87-110.

[5] Coffield, F., Learning Styles and Pedagody in post-16 learning: A systematic and critical review. Learning \& Skills research centre. http://www.lsda.org.uk/files/pdf/1543.pdf

[6] Cristea, A.I., and Calvi, L. The three Layers of Adaptation Granularity. UM'03. Springer.

[7] Cristea, A., De Mooij, A. LAOS: Layered WWW AHS Authoring Model and its corresponding Algebraic Operators. In Proceedings of WWW'03, Alternate Education track. (Budapest, Hungary 20-24 May 2003). ACM

[8] Cristea, A., and Okamoto, T., MyEnglishTeacher - A WWW System for Academic English Teaching, ICCE 2000, International Conference on Computer in Education, Learning Societies in the New Millenium: Creativity, Caring and Commitments, Taipei, Taiwan, 2000.

[9] IMS Content Packaging Specification,

http://www.imsglobal.org/content/packaging/index.cfm

[10] Julita Vassileva. Dynamic Courseware Generation on the WWW, Proceedings of the workshop "Adaptive Systems and User Modeling on the World Wide Web", Sixth International Conference on User Modeling, Chia Laguna, Sardinia, 2-5 June 1997.

[11] MOT, http://adaptmot.sourceforge.net/

[12] Stach, N., Cristea, A. \& De Bra, P. Authoring of Learning Styles in Adaptive Hypermedia: Problems and Solutions. Proc. 13th Intl WWW Conference, NY, 2004.

[13] Stewart, C., Cristea, A., Moore, A., Brailsford, T. and Ashman, H. Authoring and Delivering Adaptive Courseware, 2nd International Workshop on Authoring of Adaptive and Adaptable Educational Hypermedia, at the AH'04, Eindhoven, The Netherlands,

http://wwwis.win.tue.nl/ acristea/AH04/workshopAH.htm [14] W3C Semantic Web. http://www.w3.org/2001/sw/ 